\title{
Impact of Uncertainty Propagation on the Design of State Estimation for Hybrid ac-dc Grids
}

\author{
Abhinav Sadu, Marco Pau, Suresh Pillai, Ferdinanda Ponci, Antonello Monti \\ Institute for Automation of Complex Power Systems \\ E.ON ERC - RWTH Aachen University \\ Mathieustrasse 10, 52074 Aachen, Germany \\ Email: [asadu, mpau, spillai, fponci, amonti]@eonerc.rwth-aachen.de
}

\begin{abstract}
In order to ensure safe and reliable operation of the grids of the future, hybrid ac-dc grids are being considered as a promising solution. Traditionally state estimation (SE) of hybrid ac-dc grids has been performed with sequential SE algorithms. In this paper the authors show the inherent problems of the sequential SE algorithms, especially due to the incorrect propagation of the uncertainty of the boundary bus quantities. Instead, a two-step SE accounting for the converter losses is proposed, which updates the boundary results of the ac and dc grids respectively, to improve the accuracy. Tests of a simple hybrid ac-dc grid are presented to show the flaws in the sequential SE algorithms and the benefits of the proposed two-step method. Furthermore the accuracy improvements achievable through the inclusion of the converter losses in the $\mathrm{SE}$ algorithm are presented.
\end{abstract}

Index Terms-AC/DC grids, HVDC, Multi-Terminal DC grids, State Estimation, Uncertainty propagation

\section{INTRODUCTION}

As the energy demand and the share of generation from Distributed Energy Resources (DERs) is increasing, the traditional ac grids are often being operated at their limits. In order to effectively integrate the generation from the remote DERs and ensure a flexible, reliable and efficient operation of the grids, the use of Multi Terminal dc (MTdc) grids, on top of or in complement to the existing ac grids, is being considered as a viable solution [1]. A pan European multi terminal $\mathrm{HVdc}$ supergrid is proposed to interconnect various European countries and regions around European borders in order to share the renewable energy generation from different locations and to reduce the inefficient spinning reserve [2]. Similarly, as the distribution grids become active, MTdc grids are being considered for the integration of DERs and storage systems and to provide ac grid support for voltage and frequency control [3], [4].

With a vision that in the future the smart distribution grids would compose of hybrid ac-dc grids, research activities are being conducted at the centre for Flexible Electrical Networks (FEN) to develop planning methodologies, design, operation and control schemes for such grids [5], [6]. To operate the ac-dc grids, an accurate knowledge of the system state is key to select the appropriate control actions. Previous studies

(c) 2016 IEEE. Personal use of this material is permitted. Permission from IEEE must be obtained for all other uses. DOI: 10.1109/AMPS.2016.7602806. Publisher version: http://ieeexplore.ieee.org/document/7602806/ proposed independent or sequential State Estimation (SE) algorithms for estimating the states of hybrid ac-dc grids. For instance, in [7], a dc system state estimation is proposed without considering the ac-dc connection components. In [8] the hybrid ac-dc grid is divided into three subsystems, namely the ac, dc and ac-dc interface subsystems, and each subsystem is estimated independently. In [9] a fast decoupled sequential method is proposed, which solves iteratively the active and reactive power flows at the boundary bus between the ac and dc grids. The authors in [10] present an improved sequential method for ac-dc grid state estimation, where coupling submatrices are used to couple the ac and dc subsystems. However, to the best of our knowledge, no previous study analyzed the possible errors introduced in the estimation due to the successive iterations of a sequential SE algorithm. Furthermore, many proposals do not consider the converter losses in the SE algorithm, which can degrade the accuracy of the estimation if not duly considered.

The main focus of this paper is to compare the performance of different approaches for SE in hybrid ac-dc grids, to specifically highlight the flaws of sequential SE algorithms for hybrid ac-dc grids. Two designs of the sequential SE method and a two-step SE algorithm are presented and tested on a simple 5-bus ac network connected to an embedded 3-terminal dc network. Test results show that the uncertainty propagation in the iterations of the sequential algorithms may lead to detrimental effects, thus determining superior performance of the proposed two-step algorithm. Moreover, the impact of neglecting the converter losses is investigated, demonstrating the need to consider them in the SE model.

In the following, Section II presents the general model used to perform SE in hybrid ac-dc grids. The sequential and the two-step SE algorithms used for the analysis are elucidated in Section III. In Section IV, the performance of the aforementioned SE algorithms are presented and discussed.

\section{State Estimation Model}

$\mathrm{SE}$ is a mathematical tool used to derive the accurate knowledge of the systems states from measurements corrupted with noise. The Weighted Least Square (WLS) method is one of the most common techniques adopted for SE, thus the algorithms here presented refer to this approach. The general 
measurement model used in the WLS formulation is:

$$
\mathbf{z}=\mathbf{h}(\mathbf{x})+\mathbf{e}
$$

where $\mathbf{z}$ is the vector of measurements, $\mathbf{x}$ is the vector of state variables, $\mathbf{h}(\mathbf{x})$ are the functions linking the measurements to the state variables, and $\mathbf{e}$ is the vector of measurement errors. The measurement errors are usually considered to be uncorrelated with zero mean, whose covariance matrix has the variance of the individual measurements in the diagonal terms.

In the WLS approach, the states of the system are calculated by minimizing the following objective function:

$$
J(\mathbf{x})=[\mathbf{z}-\mathbf{h}(\mathbf{x})]^{T} \mathbf{W}[\mathbf{z}-\mathbf{h}(\mathbf{x})]
$$

where the weighting matrix $\mathbf{W}$, equal to the inverse of the measurement covariance matrix, is introduced to provide higher weight to the measurements with better accuracy.

The minimization of (2) is done iteratively, which finally results in solving the so-called normal equations:

$$
\mathbf{G} \Delta \mathbf{x}=\mathbf{H}^{T} \mathbf{W}[\mathbf{z}-\mathbf{h}(\mathbf{x})]
$$

where $\mathbf{H}$ is the Jacobian of the measurement functions $\mathbf{h}(\mathbf{x})$, $\mathbf{G}=\mathbf{H}^{T} \mathbf{W H}$ is the so-called Gain matrix, and $\Delta \mathbf{x}$ is the vector used to update the state variables $\mathbf{x}$, at each iteration $t$, using the following:

$$
\mathbf{x}_{t+1}=\mathbf{x}_{t}+\Delta \mathbf{x}
$$

The iterative process stops when a given convergence criterion is achieved, which is usually chosen as $\max (|\Delta \mathbf{x}|)<\epsilon$, where $\epsilon$ is the chosen threshold.

In hybrid ac-dc grids, three different subsystems need to be mapped into the SE model: the ac grid, the dc grid and the converter between ac and dc grid. Following subsections describe more in detail the modelling of these subsystems for SE purposes.

\section{A. Ac grid model}

The model used to represent ac grids in state estimation is well-known [11]. Traditionally, for ac transmission systems, voltage magnitudes and phase angles are considered as state variables. In such a case the resulting state vector is:

$$
\mathbf{x}_{a c}=\left[\boldsymbol{\theta}_{a c}, \mathbf{V}_{a c}\right]=\left[\theta_{2}, \ldots, \theta_{N_{a c}}, V_{1}, \ldots, V_{N_{a c}}\right]
$$

where $\theta_{i}$ and $V_{i}$ are the voltage phase-angle and magnitude of the $i$-th node, respectively, and $N_{a c}$ is the total number of nodes in the ac grid. However, it is worth remarking that hybrid ac-dc grids can be found both in transmission and distribution systems. As a result, a different choice of the state $\mathbf{x}_{a c}$ can be also adopted, without any loss of generality, taking into account the characteristics of the grid (see for example [12] or [13] for other solutions used in distribution systems).

The available measurements $\mathbf{z}_{a c}$ could be active/reactive power flows, active/reactive power injections, current flows and voltage measurements. The related expressions for the measurement functions $\mathbf{h}_{a c}$ can be found in [11] for the traditional state vector based on polar voltages.

\section{B. Dc grid model}

The dc grid can be both asymmetric (monopolar) and symmetric (monopolar or bi-polar). Nonetheless, the dc branches are simply modelled as resistive lines for steady state analysis. Similarly to ac systems, node voltages are here considered as state variables even if different options could be also possible. The resulting state vector $\mathbf{x}_{d c}$ is thus:

$$
\mathbf{x}_{d c}=\left[V_{1}, \ldots, V_{N_{d c}}\right]
$$

where $V_{i}$ is the voltage at the generic node $i$ and $N_{d c}$ is the number of nodes of the dc grid.

The possible available measurements $\mathbf{z}_{d c}$ considered for SE are: voltage magnitude, active power flows and injections, current flows and injections. The measurement function for the voltages is clearly given by the same state variables used in $\mathbf{x}_{d c}$. As for the current and power flow measurements, if the measurement in the branch between the generic nodes $i$ (sending node) and $j$ (receiving node) is considered, the following holds:

$$
\begin{gathered}
h_{I_{i j}}\left(\mathbf{x}_{d c}\right)=g_{i j} \cdot\left(V_{i}-V_{j}\right) \\
h_{I_{i j}}\left(\mathbf{x}_{d c}\right)=k \cdot g_{i j} \cdot V_{i}\left(V_{i}-V_{j}\right)
\end{gathered}
$$

where $g_{i j}$ is the conductance of the considered line, and $k$ is a coefficient equal to 1 in case of asymmetric monopolar grid or 2 in case of symmetric monopolar and bipolar grid. As for the current and power injection on the generic node $i$, they can be expressed as:

$$
\begin{gathered}
h_{I_{i}}\left(\mathbf{x}_{d c}\right)=\sum_{\substack{j=1 \\
j \neq i}}^{N_{d c}} g_{i j} \cdot\left(V_{i}-V_{j}\right) \\
h_{I_{i}}\left(\mathbf{x}_{d c}\right)=\sum_{\substack{j=1 \\
j \neq i}}^{N_{d c}} k \cdot g_{i j} \cdot V_{i}\left(V_{i}-V_{j}\right)
\end{gathered}
$$

\section{Converter model}

In hybrid grids the converters are the interface between ac and dc network. As a consequence, their accurate model is key to correctly link the ac and dc quantities. Due to the gaining popularity of Voltage Source Converters (VSC) for MTdc grids [1], in this study VSCs are considered as the connection between the ac and dc grid.

The VSC can be modelled as a controllable voltage source interfaced to the ac grid via a reactor, a capacitive filter and a transformer [14]. The schematic representation of the VSC equivalent model is depicted in Fig. 1, where the impedances of the phase reactor and transformer, and the susceptance of the filter are expressed as $Z_{c}, Z_{t f}$ and $B_{f}$, respectively. Two additional nodes are created for every connection of a converter on the ac side: firstly the filter bus, which is between the transformer and reactor, and secondly the converter bus. For the SE purposes these additional nodes are considered as a part of the ac grid and therefore the original ac grid state 


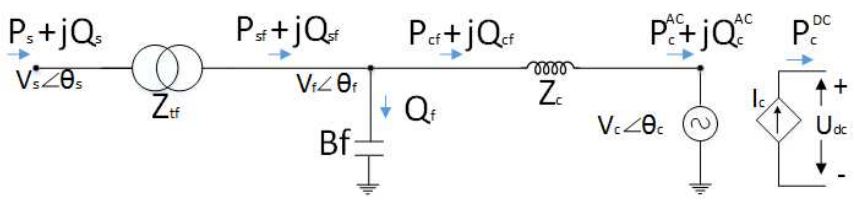

Fig. 1. Equivalent model of a converter station

vector (5) is extended to include the polar voltage quantities of the filter bus and the converter bus as shown in (11):

$$
\mathbf{x}_{a c}^{\prime}=\left[\boldsymbol{\theta}_{a c}, \boldsymbol{\theta}_{f}, \boldsymbol{\theta}_{c}, \mathbf{V}_{a c}, \mathbf{V}_{f}, \mathbf{V}_{c}\right]
$$

where $\boldsymbol{\theta}_{f}$ and $\mathbf{V}_{f}$ are the vectors containing the voltage magnitudes and phase-angles at the filter bus of each converter in the grid, and $\boldsymbol{\theta}_{c}$ and $\mathbf{V}_{c}$ are the analogous quantities at the converter bus. In a similar way, possible measurements available in the path between transformer and converter are added to the ac grid measurements to create the extended measurement vector $\mathbf{z}_{a c}^{\prime}$.

Irrespective of the type of control strategies that regulate the converter outputs, the active power flow on the ac and dc side of the converter differs only by the converter losses. Indicating with $P_{c}^{a c}$ and $P_{c}^{d c}$ these converter active powers (on the ac and dc side, respectively) and with $P_{\text {loss }}$ the above mentioned losses, the following holds:

$$
P_{c}^{d c}=P_{c}^{a c}-P_{l o s s}
$$

It is worth noting that $P_{c}^{a c}$ and $P_{c}^{d c}$ can be also seen as equivalent power injections for the ac and dc grid, respectively, and they can be deducted from the estimated state vectors (11) and (6). As for power losses $P_{\text {loss }}$, their calculation strictly depends on the specific features of the converter. In this study they are modelled as a function of the current magnitude at the ac converter bus (such current can be also computed from the estimated state vector (11)). As suggested in [14], the relationship here used for the power losses calculation is:

$$
P_{l o s s}=a+b \cdot I_{c}^{a c}+c \cdot I_{c}^{a c 2}
$$

where $I_{c}^{a c}$ is the ac side converter current magnitude, and $a$, $b$ and $c$ are loss coefficients characterizing the switching and conduction losses of the converter [14].

\section{AC-DC State Estimation Algorithms}

This Section describes the different approaches to ac-dc hybrid SE tested in this paper. The models described in Section II basically lead to two grids, ac and dc, which are linked by the boundary powers at the converters through equation (12). The basic idea of all the approaches is to perform SE independently in each grid and to use the estimated state variables to compute the respective boundary powers at the converters. In this way, the active power injections at the converter nodes of each grid are calculated. These injections are then exchanged with the other grid (taking into account the contribution of the power losses given in (12)) where they are considered as additional measurements while performing the subsequent SE step. It is worth underlining that, since each SE run is based on the WLS approach, the weights of the exchanged power injections are also to be calculated (see for example [15] for the impact of the weights on SE results). To this purpose, the uncertainty propagation law is applied by means of the following matrix multiplication:

$$
\boldsymbol{\Sigma}_{\mathbf{y}}=\left[\frac{\partial \mathbf{f}_{y}(\mathbf{x})}{\partial \mathbf{x}}\right]\left[\boldsymbol{\Sigma}_{\mathbf{x}}\right]\left[\frac{\partial \mathbf{f}_{y}(\mathbf{x})}{\partial \mathbf{x}}\right]^{T}
$$

where $\boldsymbol{\Sigma}_{\mathbf{y}}$ is the covariance matrix of the indirectly calculated quantities $\mathbf{y}, \boldsymbol{\Sigma}_{\mathbf{x}}$ is the covariance matrix of the estimated state variables $\mathbf{x}$ (which can be obtained as the inverse of the Gain matrix in (3)) and $\mathbf{f}_{y}(\mathbf{x})$ is the function linking the computed quantities $\mathbf{y}$ to the starting variables $\mathbf{x}$. The same concept of uncertainty propagation is also used at the end of the SE process to derive the theoretical uncertainty of the final estimated quantities. In this way, the SE algorithm can provide as output the estimated quantities together with their associated uncertainty.

As detailed in the following subsections, all the presented approaches share this common structure. The main differences only refer to the coordination procedure and the pre-processing operations needed before each new SE iteration.

\section{A. Sequential SE algorithms}

Fig. 2 shows the flowchart of the designed sequential SE algorithms. The procedure starts with the execution of SE in the ac grid, which uses the vector of starting measurements $\mathbf{z}_{a c}^{\prime}$. The resulting state estimates $\mathbf{x}_{a c}^{\prime}$ are then used to calculate the boundary power injections $P_{c}^{a c}$ at the converter node, the power losses $P_{\text {loss }}$ and, finally, the equivalent boundary power injection $P_{c}^{d c}$ for the dc grid. Moreover, (14) is used to calculate the corresponding uncertainties for $P_{c}^{d c}$.

In the following step, dc grid SE is performed. The equivalent power injections $P_{c}^{d c}$ provided by the ac grid are used in addition to the set of measurements $\mathbf{z}_{d c}$. Similarly to the ac grid step, the resulting state $\mathbf{x}_{d c}$ is then used to calculate the boundary powers at the converter in order to obtain the equivalent power injection $P_{c}^{a c}$ for the ac grid.

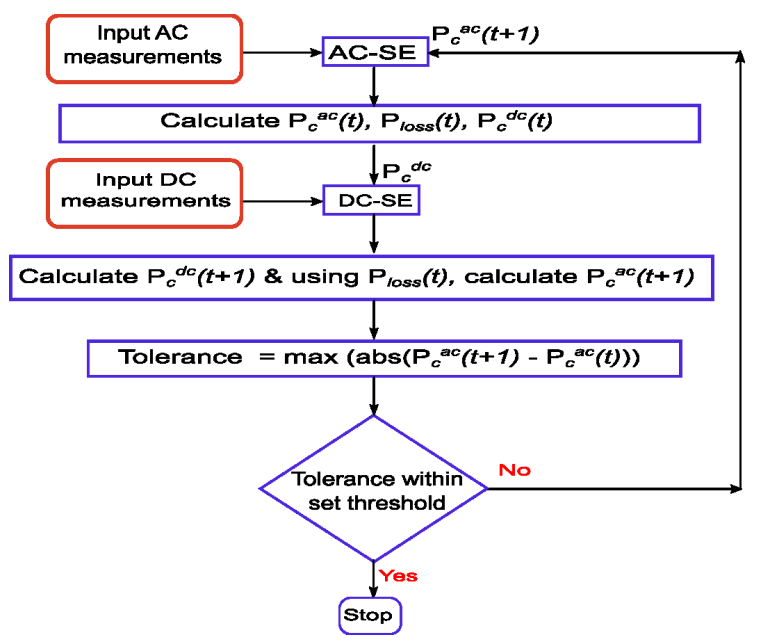

Fig. 2. Flowchart of the sequential SE algorithms 
From the second run onwards, two different options are used to continue the sequential procedure. In the first case (named in the following as "SE method 1") ac and dc grid SE are repeated iteratively using the previously estimated states as input measurements, together with the equivalent power injections $P_{c}^{a c}$ and $P_{c}^{d c}$, respectively, coming from the other grid. Such a solution is often used in multi-step SE algorithms since it allows only few iterations for the convergence. However it also implies the use of the Gain matrix as weighting matrix for the state estimates, thus meaning the introduction of a full weighting matrix in the SE process, with the consequent computational drawbacks.

To deal with this issue, in the second option (named in the following as "SE method 2"), the starting measurements $\mathbf{z}_{a c}^{\prime}$ and $\mathbf{z}_{d c}$ are again used as input to the ac and dc grids along with the additional converter power injections. This choice can be computationally less expensive, in particular in large grids, since the weighting matrix of the starting measurements always has a diagonal form.

Both SE method 1 and SE method 2 keep iterating sequentially between ac and dc grid, and exchanging the active power injection measurements at the converter, until a defined convergence threshold is achieved. Here, the chosen convergence criterion is:

$$
\max \left[\mathbf{P}_{c}^{a c}(t+1)-\mathbf{P}_{c}^{a c}(t)\right]<\epsilon
$$

where $\mathbf{P}_{c}^{a c}$ is the vector including the ac power injections at all the existing converters, $t$ indicates the iteration number and $\epsilon$ is the selected threshold.

\section{B. Two-step SE algorithm}

As alternative to the sequential algorithms, the two-step procedure proposed in [16] is here presented. Fig. 3 shows the flowchart of this two-step SE method. As it can be observed, the first step involves the parallel execution of SE for the ac and dc grids using the starting $\mathbf{z}_{a c}^{\prime}$ and $\mathbf{z}_{d c}$ measurements, respectively. Similarly to the sequential methods, the ac grid calculates from the state estimates the boundary powers $P_{c}^{a c}$ at the converter nodes, the losses $P_{\text {loss }}$, and finally the $\mathrm{dc}$ power injections $P_{c}^{d c}$ using (12). Exactly in the same way, the dc grid computes the boundary quantity $P_{c}^{d c}$ at its converter buses and sends this information to the ac grid where, using (12), the equivalent ac power injection can be deducted. The uncertainties to be considered for the WLS weights are calculated by using (14).

Once obtained the input power injection measurements for both the ac and dc grid, the second step SE can be run. To do this, the boundary quantities $P_{c}^{a c}$ and $P_{c}^{d c}$ are added to the set of starting measurements $\mathbf{z}_{a c}^{\prime}$ and $\mathbf{z}_{d c}$ (respectively) and provided as input to the second step SE. The procedure stops after each individual grid has updated its state estimates.

\section{TESTS AND RESUltS}

The presented ac-dc SE algorithms were implemented in Matlab and tested in simulation environment to assess their performance. Tests have been performed on the small hybrid

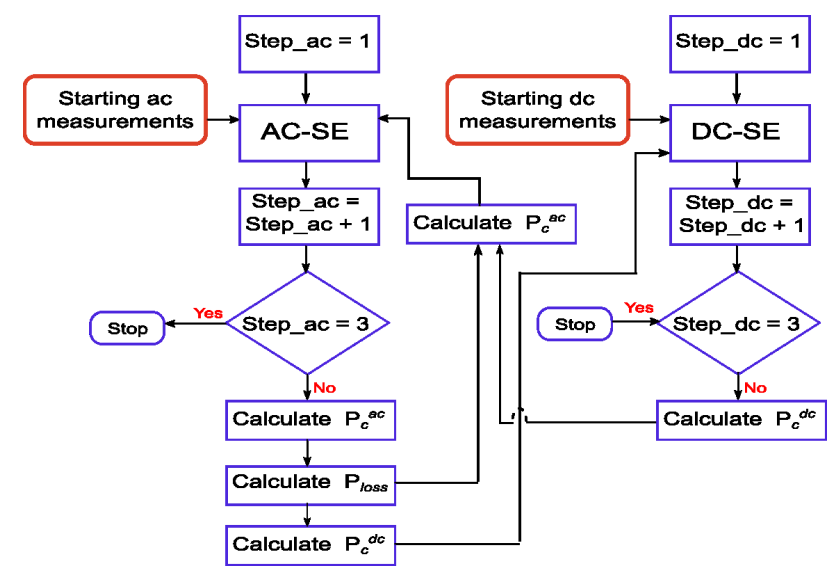

Fig. 3. Flowchart of the two-step SE algorithms

ac-dc grid depicted in Fig. 4, which is composed of a 5-bus ac grid interconnected through a multi-terminal link to a 3bus dc network. Data of the grid can be found in [17], which also provides the tool for running the power flow analysis duly taking into account the converter power losses. Tests were carried out considering the power flow results as "true" reference conditions of the network and then extracting the selected measurements by perturbing the reference quantities with random noise, according to the uncertainty level assumed for each measurement. The measurement configuration used for the tests includes:

- Ac grid: voltage magnitude measurements at nodes 4 and 5, and active and reactive power flow measurement for each branch. In addition, power flow measurements for all the converters, both in the transformer and in the reactor branch, and zero injection knowledge at the filter bus (between transformer and reactor) are considered.

- Dc grid: voltage measurement at bus 1, current measurement in the branch between nodes 2 and 3, and power measurement in the line between nodes 1 and 3 .

All the measurements were considered to have Gaussian distributed error with standard deviation equal to one third of the assumed value of uncertainty. Simulations with 25000 Monte Carlo (MC) trials were used to statistically analyze the $\mathrm{SE}$ results and to assess the goodness of the theoretical uncertainty provided as output by the different hybrid SE approaches.

The first series of tests has been conducted to compare the performance of the different SE methods. A first drawback found while performing the tests is the necessity to suitably tune the convergence threshold of the sequential SE algorithms depending on the uncertainty considered for the measurement devices. Once this is done, in general, all the implemented algorithms exhibit similar performance in the ac and dc grid. Fig. 5 shows as an example the Root Mean Square Error (RMSE) results obtained for the estimation of the voltage magnitude on the dc grid nodes when a measurement uncertainty equal to $1 \%$ is considered for all the measurements (both ac and dc). It is possible to observe that all the approaches allow improving the estimation accuracy with respect to the case in which the 


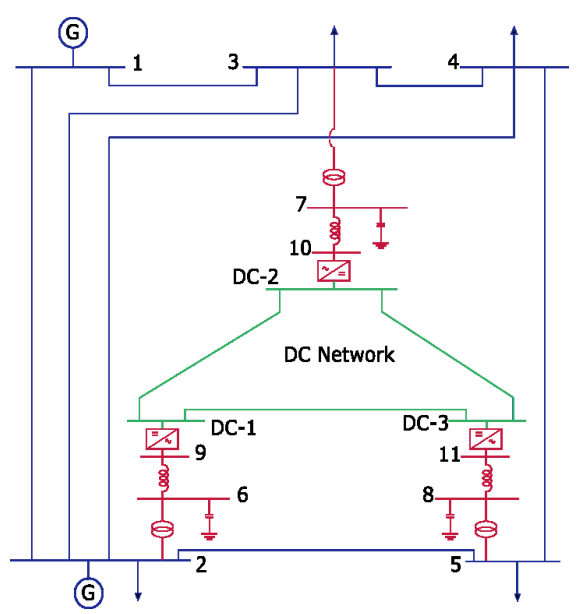

Fig. 4. Ac-dc test network

only dc grid SE is performed. But, in particular, it is worth underlining that the two-step algorithm, despite using less SE iterations, leads to the same improvements as the sequential methods.

The capability of the different approaches to provide the correct theoretical uncertainty (using the uncertainty propagation law as shown in (14)) has also been tested. In this case, different results were found for the tested algorithms. In particular, the sequential SE approaches proved to be unable to correctly provide the theoretical uncertainty of the estimated quantities, while very good results were found for the twostep SE method. Table I shows the results achieved for SE method 1 (similar results also hold for SE method 2) in terms of expanded uncertainty (considering a coverage factor equal to 3) expressed as percentage with respect to the dc bus voltage values. In the Table, the statistical uncertainty obtained through the MC test is compared to the theoretical uncertainties provided as output from the estimator. Since a value of theoretical uncertainty is obtained for each MC trial (for each monitored quantity), Table I reports the minimum, average and maximum values of theoretical uncertainty obtained during the MC simulation (for the dc bus voltage estimates). From such results it is evident that the statistically expected uncertainty and the theoretical uncertainty do not match. Table II reports instead the analogous results obtained for the two-step SE algorithm. It is clear that in this case

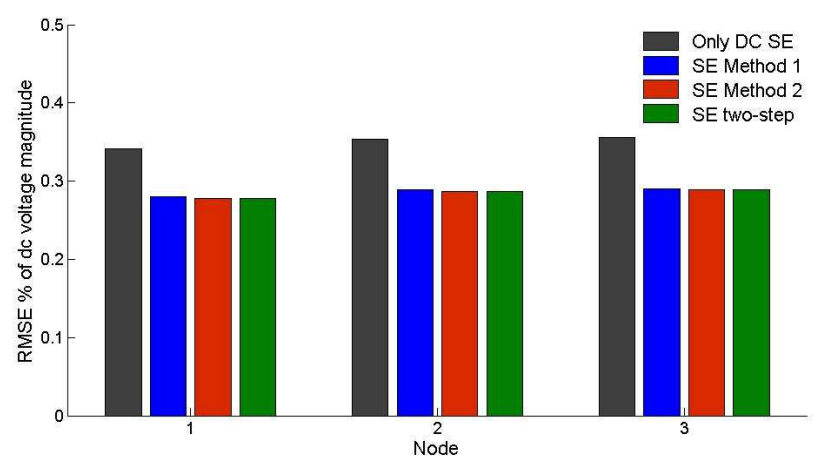

Fig. 5. RMSE of dc grid voltage magnitude estimates
TABLE I

STATISTICAL VS THEORETICAL UNCERTAINTY FOR SE METHOD 1

\begin{tabular}{|c|c|c|c|c|}
\hline Dc voltage & Monte Carlo & \multicolumn{3}{|c|}{ Theoretical uncertainty [\%] } \\
\cline { 3 - 5 } estimation & uncertainty [\%] & Minimum & Average & Maximum \\
\hline dc bus 1 & 0.83 & 0.06 & 0.11 & 0.36 \\
\hline dc bus 2 & 0.86 & 0.07 & 0.11 & 0.36 \\
\hline dc bus 3 & 0.86 & 0.07 & 0.11 & 0.37 \\
\hline
\end{tabular}

TABLE II

STATISTICAL VS THEORETICAL UNCERTAINTY FOR TWO-STEP SE

\begin{tabular}{|c|c|c|c|c|}
\hline \multirow{2}{*}{$\begin{array}{c}\text { De voltage } \\
\text { estimation }\end{array}$} & Monte Carlo & \multicolumn{3}{|c|}{ Theoretical uncertainty [\%] } \\
\cline { 3 - 5 } & uncertainty [\%] & Minimum & Average & Maximum \\
\hline dc bus 1 & 0.83 & 0.82 & 0.83 & 0.83 \\
\hline dc bus 2 & 0.86 & 0.85 & 0.85 & 0.86 \\
\hline dc bus 3 & 0.86 & 0.85 & 0.86 & 0.86 \\
\hline
\end{tabular}

a really reliable computation of the theoretical uncertainty is instead obtained.

Fig. 6 shows the trend of the dc bus voltage estimates, together with their theoretical uncertainty, during the different iterations of the sequential SE method. It is possible to observe the trend of the estimated values stabilizes immediately , while the calculated theoretical uncertainties decrease at each iteration. This explains the varying limits of theoretical uncertainty obtained in Table I: in fact, during the different MC trials, a different number of iterations can be needed by the sequential SE algorithm to converge. The reason for this misbehaviour of the sequential algorithms is likely due to the presence of neglected correlations between the internal grid inputs and the converter power injections acquired from the other grid. In fact, at each new SE iteration of the sequential algorithm, the converter power injections are considered as totally independent with respect to all the other inputs (no mutual correlations in the weighting matrix). This can lead to overestimate their impact on the estimation results if some correlations actually exist. The same problem is also mentioned in [18] where it is highlighted that neglecting the mutual covariance between internal and boundary variables in multi-area SE approaches can lead to poor SE solutions. In the sequential approach, the presence of these correlations is likely, since the power injections used in the subsequent steps are previously processed by the same SE algorithm. It is worth

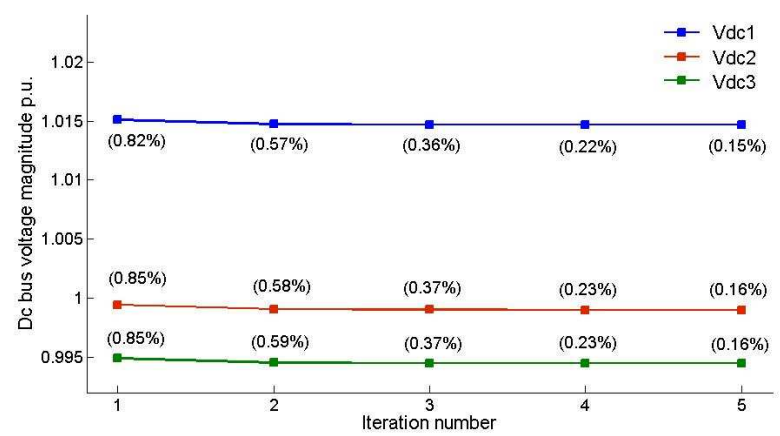

Fig. 6. Dc bus voltage estimates over sequential SE iterations with indication of the theoretical uncertainty (between parenthesis) 


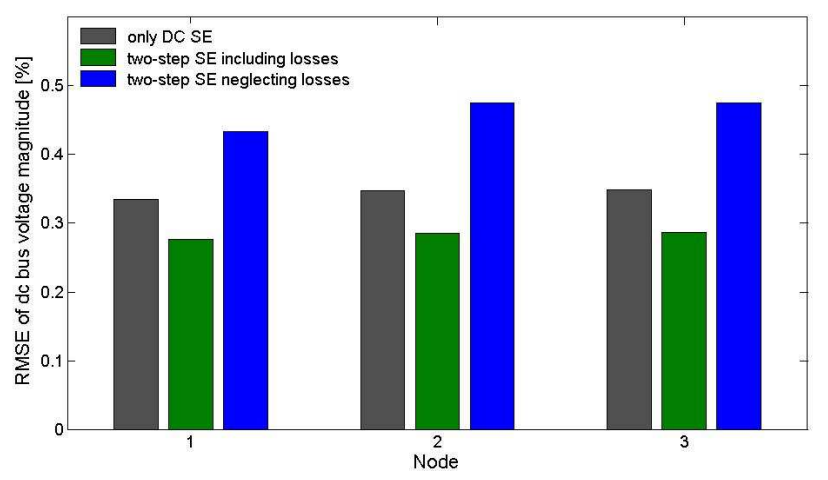

Fig. 7. RMSE of dc grid voltage magnitude estimates when including or neglecting converter power losses

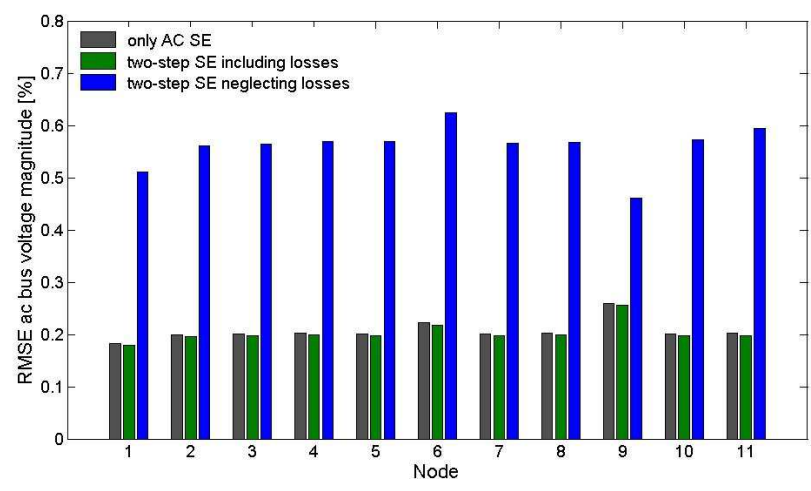

Fig. 8. RMSE of ac grid voltage magnitude estimates when including or neglecting converter power losses

noting that the same problem does not appear in the two-step SE algorithm, since in this case the converter power injections used at the second step have not been previously processed by the same grid, and thus they are of course independent with respect to the other internal inputs.

The impact of considering the converter losses in the SE approach has also been evaluated. The results shown here refer to the two-step SE algorithm and to the same measurement configuration used in the previous tests. Fig. 7 shows, as an example, the RMSE results obtained for the dc bus voltage estimates, while Fig. 8 shows the same results for ac grid bus voltages. It is evident that neglecting the converter losses can drastically affect the accuracy of the estimation results, leading to poor SE results even when an accurate measurement system is deployed on the grid.

\section{CONCLUSIONS}

This paper has analyzed the performance of different types of hybrid ac-dc grid SE design. Two main conclusions can be drawn by the performed analysis. Firstly, the iterative procedure in the sequential SE approach does not allow improving the estimation results. Consequently, a two-step approach is preferable since it requires fewer iterations and computation time. Moreover, the correct propagation of the uncertainty, needed to obtain the theoretical uncertainties of the estimates, can be only achieved through the two-step algorithm. It is important remarking that the capability to provide the theoretical uncertainty as output of the SE process is a very important feature of the WLS formulation. This information can be in fact exploited from the upper level control applications, for example to properly select the most appropriate safety margins to be used. Finally, performed studies clearly show the importance to take into account the converter power losses in the design of the ac-dc SE algorithm.

\section{ACKNOWLEDGEMENTS}

This work has been funded by the Bundesministerium für Forschung und Bildung, Forschungscampus Elektrische Netze der Zukunft (FKZ: 03SF0490).

\section{REFERENCES}

[1] D. Van Hertem, O. Gomis-Bellmunt, and J. Liang, HVDC Grids: For Offshore and Supergrid of the Future. John Wiley \& Sons, Inc., 2016.

[2] H. Ergun, J. Beerten, and D. V. Hertem, "Building a new overlay grid for Europe," in 2012 IEEE Power and Energy Society General Meeting, July 2012 , pp. $1-8$.

[3] D. J. Hammerstrom, "AC versus DC distribution systems - Did we get it right?" in Power Engineering Society General Meeting, 2007. IEEE, June 2007, pp. 1-5.

[4] A. Mohamed, V. Salehi, and O. Mohammed, "Reactive power compensation in hybrid AC/DC networks for smart grid applications," in 2012 3rd IEEE PES Innovative Smart Grid Technologies Europe (ISGT Europe), Oct 2012, pp. 1-6.

[5] Center for flexible electrical networks. [Online]. Available: http: //www.fen.rwth-aachen.de/

[6] A. Korompili, A. Sadu, F. Ponci, and A. Monti, "Flexible electric networks of the future: Project on control and automation in MVDC grids," in International ETG Congress 2015; Die Energiewende - Blueprints for the new energy age; Proceedings of, Nov 2015, pp. 1-8.

[7] J. D. Glover and M. Sheikoleslami, "State estimation of interconnected HVDC/AC systems," IEEE Power Engineering Review, vol. PER-3, no. 6, pp. 55-56, June 1983.

[8] A. M. L. da Silva, G. Perrotta, R. B. Prada, and D. M. Falcao, "State estimation for integrated multi-terminal DC/AC systems," IEEE Power Engineering Review, vol. PER-5, no. 9, pp. 31-31, Sept 1985.

[9] R. Jegatheesan and K. Duraiswamy, "AC multiterminal DC power system state estimation - a sequential approach," Electric Machines \& Power Systems, vol. 12, no. 1, pp. 27-42, 1987.

[10] Q. Ding, T. S. Chung, and B. Zhang, "An improved sequential method for AC/MTDC power system state estimation," IEEE Transactions on Power Systems, vol. 16, no. 3, pp. 506-512, Aug 2001.

[11] A. Abur and A. G. Expòsito, Power System State Estimation. Theory and Implementation. Marcel Dekker, New York, 2004.

[12] M. Pau, P. A. Pegoraro, and S. Sulis, "Efficient branch-current-based distribution system state estimation including synchronized measurements," IEEE Trans. Instrum. Meas., vol. 62, no. 9, pp. 2419-2429, Sep. 2013.

[13] M. Pau, P. A. Pegoraro, and S. Sulis, "Performance of three-phase WLS distribution system state estimation approaches," in Applied Measurements for Power Systems (AMPS), 2015 IEEE International Workshop on, Sept 2015, pp. 138-143.

[14] J. Beerten, S. Cole, and R. Belmans, "Generalized steady-state VSC MTDC model for sequential AC/DC power flow algorithms," IEEE Transactions on Power Systems, vol. 27, no. 2, pp. 821-829, May 2012.

[15] M. Asprou, E. Kyriakides, and M. Albu, "The effect of variable weights in a wls state estimator considering instrument transformer uncertainties," IEEE Trans. Instrum. Meas., vol. 63, no. 6, pp. 1484 1495, Jun. 2014.

[16] M. Pau, A. Sadu, S. Pillai, F. Ponci, and A. Monti, "A state estimation algorithm for hybrid AC/DC networks with multi-terminal DC grid," submitted to IEEE PES Innovative Smart Grid Technologies, (ISGT Europe), 2016.

[17] MatACDC sotware. [Online]. Available: http://www.esat.kuleuven.be/ electa/teaching/matacdc/

[18] A. Gomez-Exposito, A. Abur, A. de la Villa Jaen, and C. Gomez-Quiles, "A multilevel state estimation paradigm for smart grids," Proceedings of the IEEE, vol. 99, no. 6, pp. 952-976, June 2011. 